\title{
Combined 30-degree bevel up and down technique against 0 -degree phaco tip for phacoemulsification surgery of hard cataracts
}

This article was published in the following Dove Press journal:

Clinical Ophthalmology

6 June 2017

Number of times this article has been viewed

Rajesh Subhash Joshi

Sonal Jayant Muley

Department of Ophthalmology, Vasantrao Naik Government Medical College, Yavatmal, Maharashtra, India
Purpose: To evaluate the effective phaco time (EPT), cumulative dissipated energy (CDE) and nucleus emulsification time (NET) as phaco parameters with 0 - and 30-degree phaco tip.

Patients and methods: This prospective, interventional and observational case series included 294 patients scheduled for cataract removal by phacoemulsification technique. Patients with nuclear cataracts of grade 4 and 5 nuclear opalescence of Lens Opacities Classification System III were included in the study. Patients were consecutively allocated to phaco chop technique with 0 -degree (0-degree group, $\mathrm{n}=147$ ) and combined bevel up and down position using 30-degree phaco tip (combined bevel up/down group, $n=147$ ). The 0 -degree group had phacoemulsification with 0-degree phaco tip, while the 30-degree group had chopping of the nucleus with bevel down phaco tip and emulsification of nuclear fragments was accomplished with bevel up tip. EPT, CDE, NET and intraoperative complications were noted and compared between the groups using analysis of variance.

Results: Average EPT, CDE and NET were lower in the 30-degree group than in the 0-degree group. However, no statistically significant difference was found in EPT $(P=0.0733)$, $\operatorname{CDE}(P=0.0663)$ and NET $(P=0.0633)$ between the two groups. No serious intraoperative complications were noted. The anterior chamber was maintained throughout the procedure in both groups. No patients had wound burn and miosis during the procedure. None of the patients developed bullous keratopathy, uveitis and cystoid macular edema during the follow-up period.

Conclusion: Combined bevel up and down 30-degree tip can yield effective hard nucleus phacoemulsification. Bevel down tip of 30-degree helps in effective chopping and bevel up tip assists in emulsification of the nuclear fragments. Although combined bevel up and down 30-degree tip can yield effective hard nucleus phacoemulsification, no statistically significant difference was found in EPT, CDE and NET between the two groups.

Keywords: phacoemulsification, bevel up and bevel down phaco needle, effective phaco time, cumulative dissipated energy

\section{Introduction}

Phacoemulsification is a proven surgical technique for cataract surgery. For fast postoperative visual recovery and excellent visual outcome, ultrasound (US) energy should be concentrated on the lens while protecting other ocular tissues. ${ }^{1}$

Hard nuclear cataracts exist in many people, especially in Asian rural population. ${ }^{2,3}$ Phacoemulsification of hard cataracts is a challenging situation. Phaco tips varying from 0 to 45 degrees are utilized for phacoemulsification of different nuclear grades of cataracts. 
Techniques to emulsify the nucleus vary from surgeon to surgeon. 0-degree phaco tip is commonly utilized for chopping technique. However, it produces turbulence in the anterior chamber during emulsification of the nuclear fragments.

US energy in bevel up position with 30-degree phaco tip may not effectively chop the nucleus of hard grades. However, bevel up tip can emulsify nuclear fragments effectively. Bevel down tip because of better contact with the nucleus can chop the nucleus effectively and produce less turbulence. Therefore, the present study was designed to analyze the intraoperative performance of combined bevel up and bevel down 30-degree and 0-degree phaco tip in the phacoemulsification of the hard cataracts.

\section{Materials and methods}

\section{Sample size}

To assess a difference of $15 \%$ between two groups with power of $80 \%$ and an alpha error of $5 \%$ and considering $10 \%$ lost to follow-up, a sample size of 147 was considered in each group.

\section{Patient selection and study design}

The present study adhered to the tenets of Declaration of Helsinki, and ethical approval was given by the medical ethics committee of Government Medical College and Hospital, Yavatmal, India and written informed consent was obtained from all the participants. The study duration was 1 year (2014-2015).

This prospective, interventional, observational and nonrandomized case series included 294 patients with senile cataracts scheduled for phacoemulsification and intraocular lens (IOL) implantation. Patients having operable cataracts in the grade of 4 and 5 of Lens Opacities Classification System III (LOCS III) were included in the study. ${ }^{4}$ Exclusion criteria were corneal degeneration, dystrophies and scarring, glaucoma, uveitis, previous intraocular surgeries, subluxated cataracts, monocular patients, poor pupillary dilation (dilatation of pupil less than $5 \mathrm{~mm}$ ) and extremely shallow anterior chamber. Preoperative assessment included bestcorrected visual acuity (BCVA), slit lamp examination, intraocular pressure, retinal evaluation and A-scan biometry for IOL power calculation.

Non-steroidal anti-inflammatory drops (Nevanac; Alcon Laboratories, Bengaluru, India) were instilled into the eye scheduled for the surgery a day prior and on the day of surgery.

Patients were divided into two groups. The 0 -degree group $(n=147)$ had phacoemulsification with 0 -degree phaco tip, while the 30-degree combined bevel up/down group ( $n=147)$ had chopping of the nucleus with bevel down phaco tip and emulsification of nuclear fragments was accomplished with bevel up tip (Figures 1 and 2A-C). The size of the tip was $20 \mathrm{G}$. Patients were consecutively assigned to both groups.

\section{Surgical technique}

A single surgeon performed all the surgeries (R.S.J.). Preoperative dilatation of the pupil was achieved using a combination of $0.8 \%$ tropicamide and 5\% phenylephrine. Patients were operated under $0.5 \%$ topical proparacaine hydrochloride drops instilled twice $10 \mathrm{~min}$ before the surgical procedure supplemented by $0.5 \mathrm{~mL}$ subconjunctival injection of $2 \%$ lignocaine hydrochloride at the beginning of the surgery. A side port incision was created on the appropriate side as required. Trypan blue dye was used to stain anterior capsule in patients with white cataract. Viscoelastic agent (2\% hydroxypropyl methyl cellulose, Appavisc; Appasamy Ocular Devices, Puducherry, India) was injected through the side port with $23 \mathrm{G}$ blunt tip cannula. A $2.8 \mathrm{~mm}$ clear corneal temporal incision was performed. The rhexis marker was kept on the anterior surface of the cornea to delineate $5.5 \mathrm{~mm}$ area. Continuous curvilinear capsulorhexis was completed using capsulorhexis forceps under viscoelastic agent. The size of the rhexis was kept approximately 5.5-6 mm. Hydrodissection was performed with balanced salt solution (BSS).

The phacoemulsifier used during the study period adopts a conventional mode of phacoemulsification (I-master phacoemulsifier system; Appasamy Associates, Chennai, India).

The settings for the nucleus chop were power $90 \%$ (linear), vacuum $350 \mathrm{mmHg}$ and aspiration flow rate

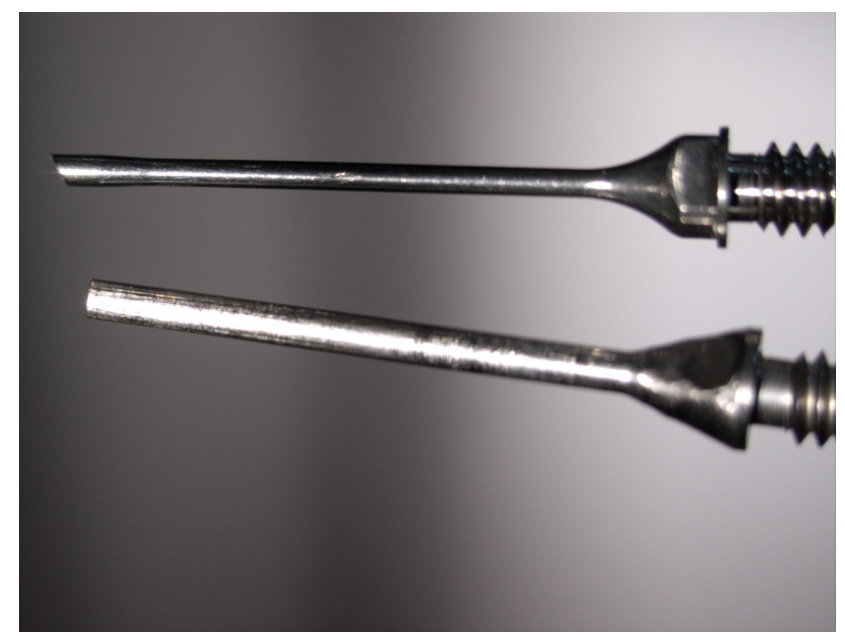

Figure I 30- and 0-degree phaco tips. 

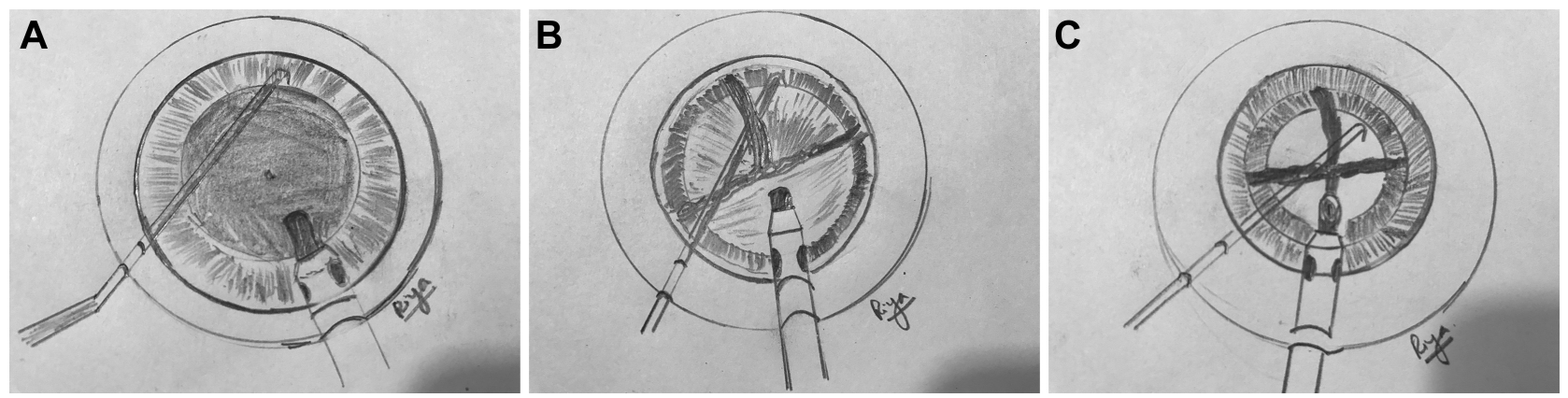

Figure 2 Illustrations of bevel down tip (A), nucleus division with bevel down tip (B), and nucleus emulsification with bevel up tip (C). Note: Images courtesy of Riya.

$34 \mathrm{~mL} / \mathrm{min}$. Cold BSS was used in all cases. Parameters were same for all the cases and were not changed till the last fragment was emulsified. Phacoemulsification was performed in the capsular bag. Quick chop technique was utilized for all cases. ${ }^{5}$ Thorough cortical cleanup was accomplished by irrigation and aspiration probe. A single-piece hydrophilic lens was implanted in the capsular bag (RYCF; Care Group, Vadodara, India). Stromal hydration of side port and main incision was completed with BSS. Patients having wound burn were tested for leakage with fluorescein strip.
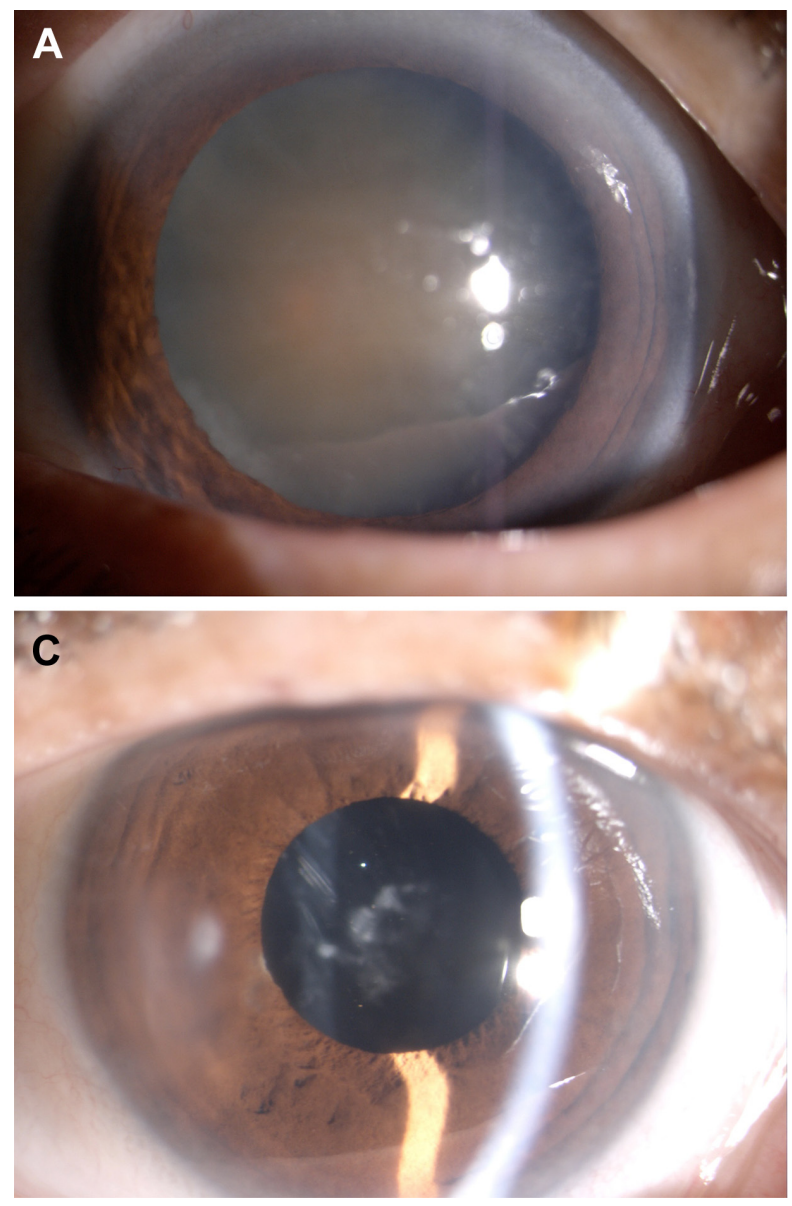

\section{Postoperative treatment}

Patients were treated with a schedule of $0.3 \%$ moxifloxacin and $1 \%$ prednisolone acetate eye drops that were tapered over 4 weeks. Non-steroidal anti-inflammatory drops were continued twice a day for 1 month. Two percent homatropine drops were instilled twice a day for 10 days.

Postoperative visits were scheduled on day 1 and 7 and at 4 weeks. Follow-up included visual outcome and slit lamp biomicroscopic examination. Preoperative and postoperative follow-up is depicted in Figure 3A-D.
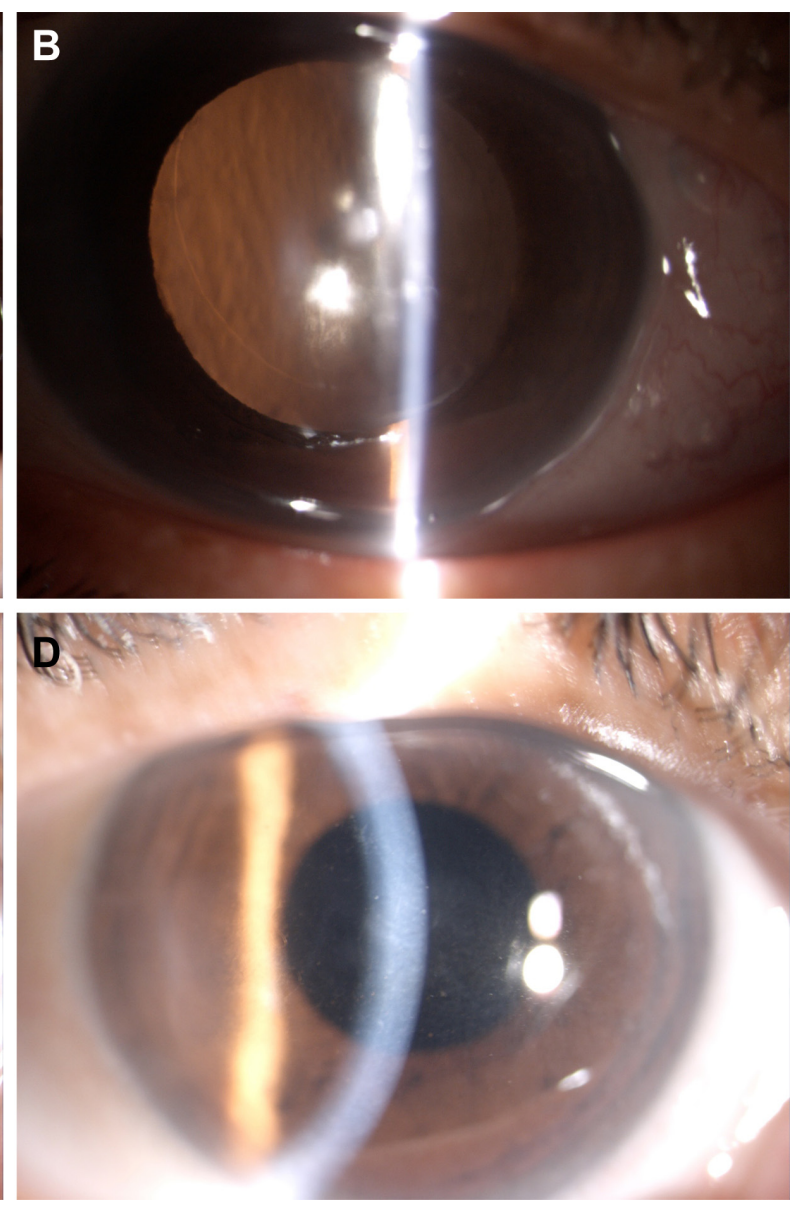

Figure 3 Preoperative grade 4 cataract (A). Slit lamp images taken immediately after operation (B), 2 weeks postoperatively (C), and 4 weeks postoperatively (D). 


\section{Intraoperative parameters studied}

The main intraoperative phaco parameters studied were effective phaco time (EPT), cumulative dissipated energy (CDE), nucleus emulsification time (NET) and intraoperative surgical complications. Beginning of the US energy till the emulsification of the last nuclear fragment was taken as NET. NET characterizes the time required for the emulsification of the nucleus. CDE represents total amount of energy at the incision. $\mathrm{CDE}$ for phaco was calculated as: $\mathrm{CDE}=$ average $\mathrm{U} / \mathrm{S}$ power $\times \mathrm{U} / \mathrm{S}$ time.

EPT signifies how many seconds the foot pedal remained in the third position.

\section{Statistical analysis}

Preoperative and intraoperative observations were entered in an Excel sheet. The data were analyzed using analysis of variance (ANOVA) and SPSS software (13.0, SPSS Inc., Chicago, IL, USA). Group differences in parameters were tested using one-way ANOVA. Multiple comparisons were tested using the Tukey test. Differences were considered significant when the $P$-value was less than 0.001 .

\section{Results}

The average age of the study patients was 69.42 years $( \pm 12.05)$. Table 1 depicts the demographic characteristics of both groups. A total of $53.4 \%$ patients had nuclear opalescence (NO) grade 4 , while $46.6 \%$ patients had NO grade 5 .

Intraoperative data regarding phaco parameters (EPT, CDE and NET) are illustrated in Table 2.

Average EPT, CDE and NET were lower in the 30-degree group than in the 0 -degree group. However, no statistically significant difference was seen in both the groups.

Intraoperative complications are shown in Table 3.

The anterior chamber was maintained throughout the procedure in both groups. No patients had wound burn and miosis during the procedure. Two patients had posterior capsular rupture (PCR) and these required sutures at the incision site. The mean postoperative visual acuity between the

Table I Demographic characteristics of both groups

\begin{tabular}{llll}
\hline Characteristic & $\begin{array}{l}\text { 0-degree } \\
\text { group }\end{array}$ & $\begin{array}{l}\text { Combined bevel } \\
\text { up/down group }\end{array}$ & P-value \\
\hline Mean age + SD (years) & $70.22 \pm 9.526$ & $70.62 \pm 14.86$ & 0.780 \\
Age range (years) & $49-90$ & $50-89$ & \\
Sex (female/male) & $87 / 60$ & $77 / 70$ & 0.568 \\
Eye (right/left) & $79 / 68$ & $100 / 47$ & \\
Cataract grade & $77 / 70$ & $80 / 67$ & 0.469 \\
(NO 4/NO 5) & & & \\
\hline
\end{tabular}

Abbreviations: NO, nuclear opalescence; SD, standard deviation.
0 -degree group and 30-degree group on day 1 was $0.45 \pm 0.12$ $\log$ MAR versus $0.42 \pm 0.11 \log$ MAR, $P=0.0667$ and at 7 and 30 days was $0.35 \pm 0.13 \log$ MAR versus $0.34 \pm 0.11 \log$ MAR, $P=0.0677$ and $0.12 \pm 0.1 \log$ MAR versus $0.11 \pm 0.1 \log$ MAR, $P=0.0778$, respectively.

The refractive status in both groups is depicted in Table 4.

During postoperative follow-up period, none of the patients developed bullous keratopathy, uveitis and cystoid macular edema.

\section{Discussion}

Surgeons performing phacoemulsification focus on minimal use of US energy and shorten the phaco time in hard grades of cataracts. The density of the nucleus determines the amount of US energy required for the emulsification of the nuclear material. Most complications of phacoemulsification result from US energy and power. ${ }^{6,7}$ Prolonged phaco time and US energy in the anterior chamber are associated with delayed postoperative corneal recovery and final visual outcome. Modulations in the phaco power have been developed to reduce the risk of thermal energy and to increase efficiency. ${ }^{8}$ Torsional mode of phacoemulsification has been shown to be effective in managing hard grades of cataracts. ${ }^{9}$ The side-to-side stroke has a cutting effect that induces no repulsion and makes the torsional phacoemulsification effective in dense nuclei. This will reduce the US time and CDE, ultimately reducing the corneal endothelial cell loss. Every ophthalmic surgeon in the developing countries may not have access to this modality due to the cost factor.

We described here a simple method for effective emulsification of hard grade of cataracts without changing the phaco parameters, technique and machine.

A pilot study conducted by us on routine cataract cases in the grade of 4 and 5 of LOCS III by phacoemulsification technique has shown sideways cutting action with 30-degree phaco tip, which was absent on 0-degree tip. Sideways cutting action was appreciated when the phaco tip was facing the nuclear fragment at an angle of 20-30 degrees. The 30-degree tip in bevel down position also showed quick build-up of rise time.

The mean effective US time in the 30-degree tip group $2.097( \pm 0.8954)$ was lower than in the 0-degree group, $2.298( \pm 0.6415)$. However, no statistically significant difference was seen between the two groups $(P=0.0733)$. The US power was $90 \%$ in linear mode for all cases. The lower US time in the 30-degree tip group could be due to effective nuclear division by the bevel down tip. Quick occlusion of the phaco tip was observed after placing the bevel down tip 
Table 2 Intraoperative parameter in both groups

\begin{tabular}{llll}
\hline Parameter & 0-degree group & $\begin{array}{l}\text { Combined bevel } \\
\text { up/down group }\end{array}$ & P-value \\
\hline Mean effective US time $( \pm S D)$, min & $2.298( \pm 0.6415)$ & $2.097( \pm 0.8954)$ & 0.0733 \\
Mean nucleus emulsification time $( \pm S D)$, min & $3.374( \pm 0.8729)$ & $2.402( \pm 3.066)$ & 0.0633 \\
Mean cumulative dissipated energy $( \pm S D)$ & $3.448( \pm 0.962)$ & $3.135( \pm 1.343)$ & 0.0663 \\
\hline
\end{tabular}

Abbreviations: US, ultrasound; SD, standard deviation.

perpendicular to the nucleus. Quick occlusion of the phaco tip was observed after placing the bevel down tip perpendicular to the nucleus, which meant fast attainment of the preset vacuum and quick chop of the nucleus was possible. The side of the phaco tip helps in cutting the nucleus. Turning the bevel up helps in effective emulsification of nuclear fragments. The resultant phaco energy, time and the NET were reduced.

Beginning of the US energy till the emulsification of the last nuclear fragment was taken as NET. The study deals with the performance of the phaco tip in up and down position; therefore, NET was appropriate in this situation. On Medline search, there were no studies on NET in bevel up and down position of the phaco tip. However, Raskin et al calculated total surgical time in the study on effect of bevel position on corneal endothelium after phacoemulsification. ${ }^{10}$ NET in both the groups of our study was less than that in the study conducted by Raskin et al.

We used phaco chop technique with sharp chopper in both the groups. The sharp chopper helps in cutting the nucleus when placed perpendicular to the nucleus. Phaco chop has been shown to be associated with reduced phaco time and power requirement because of fewer surgical maneuvers. ${ }^{11}$ It is also associated with less intraoperative complications as shown by Park et al. ${ }^{11}$

Intraoperative complications in our study were not different in both the groups. Iris catch away from the sphincter was seen in one patient in the 30-degree group. It occurred while emulsifying the last nuclear fragment that came out of the capsular bag and lodged on the peripheral part of the iris.

The capsulorhexis was kept on the larger side (approximate size $5.5-6 \mathrm{~mm}$ ). There are chances of split in the rhexis margin if the rhexis is small $(5.5 \mathrm{~mm}$ and less) with

Table 3 Intraoperative complications

\begin{tabular}{lll}
\hline Complication & 0-degree group & $\begin{array}{l}\text { Combined bevel } \\
\text { up/down group }\end{array}$ \\
\hline Posterior capsule rupture & $\mathrm{I}$ & $0 \mathrm{I}$ \\
Iris sphincter tear & 0 & $0 \mathrm{I}$ \\
Anterior capsule tear & $\mathrm{I}$ & $0 \mathrm{I}$ \\
Iris catch & 0 & $0 \mathrm{I}$ \\
\hline
\end{tabular}

subsequent extension to the posterior capsule. This increases the chances of nucleus drop. One patient each in both the groups had split in the anterior capsule, which we realized at the end of the surgery. In these patients, we could manage the placement of the IOL in the bag, as there was no extension of the rhexis to the posterior capsule. We think $6 \mathrm{~mm}$ size rhexis may avoid this complication in the phacoemulsification of the hard nuclei.

One patient in each group had PCR. Epinucleus acts as a barrier between the phaco tip and the posterior capsule and prevents PCR. In hard grade of cataracts, there is minimal or no epinucleus and the chance of PCR is enhanced. One patient had a posterior capsular plaque. While performing polishing and removal of the plaque, the posterior capsule gave way.

There was no vitreous loss. We could manage the placement of IOL in the bag after posterior capsulorhexis. One patient had PCR during removal of the last nuclear fragment. No fragment drop occurred. After thorough anterior vitrectomy, IOL was placed over the anterior capsular rim. These two patients required sutures at the incision site.

Mean CDE in the 30-degree group (3.135 \pm 1.343$)$ was less than in the 0-degree group (3.448 \pm 0.962$), P=0.0663$.

Less CDE in phacoemulsification of the cataract translates to less US energy use and is considered better for corneal recovery. ${ }^{12-16}$ High CDE reading is equated with statistically significant corneal endothelial cell loss with one surgeon, one technique and one setting. ${ }^{1718}$ Fakhry and El Shazly have shown that with an increase in the density of the nuclei there is an increase in the $\mathrm{CDE} .{ }^{19} \mathrm{CDE}$ in our study was less than the longitudinal and the torsional modality in the study by Fakhry and El Shazly. This is also reflected on to the

Table 4 Refractive status in groups

\begin{tabular}{lll}
\hline Refraction & $\begin{array}{l}\text { 0-degree } \\
\text { group (diopter) }\end{array}$ & $\begin{array}{l}\text { Combined bevel } \\
\text { up/down group (diopter) }\end{array}$ \\
\hline Preoperative sphere & $\mathrm{I} .25( \pm 0.75)$ & $\mathrm{I} .00( \pm 0.50)$ \\
Preoperative cylinder & $0.50( \pm 0.50)$ & $0.50( \pm 0.50)$ \\
Postoperative sphere & $0.50( \pm 0.75)$ & $0.50( \pm 0.50)$ \\
Postoperative cylinder & $0.25( \pm 0.50)$ & $0.25( \pm 0.50)$ \\
\hline
\end{tabular}


wound site thermal injury. No patients in our study group had wound burn. Use of cold BSS may have a role to play to reduce the corneal burn.

Joo and Kim have also shown that US time for emulsification of hard cataract by torsional mode of phacoemulsification was not significant when compared to conventional mode of phacoemulsification. The explanation offered was that US power in torsional mode does not act on the nucleus perpendicularly contrary to the conventional mode. ${ }^{20}$

Bevel down tip has been shown to cause less turbulence of the lens fragments and a better contact between the lens fragment and the phaco tip..$^{21,22}$ It has also been shown to reduce the endothelial damage because it faces away from the corneal endothelium during phacoemulsification preserving endothelium. Because of non-availability of the specular microscope during the study period, we could not study the effect of bevel position on the endothelium.

The mean BCVA was similar between the two groups at each follow-up.

We think that the technique of bevel down and up phaco requires little experience. No special skill is required and the surgeon does not need to change their phacoemulsification technique or their existing machine to learn the technique.

The surgeon was trained in chop technique; therefore, comparison with divide and conquer or other non-chop technique was not possible.

We could not compare our findings of combined bevel position in the phacoemulsification of the hard grade cataract; however, as to the best of our knowledge there were no studies available in the peer-reviewed journals. A single surgeon was involved, which does not allow comparison of skills and surgical technique. Another drawback of the study was the non-randomized nature of the study. We recommend multicenter trial with different techniques in different tip positions.

\section{Conclusion}

Cataract surgery by phaco technique in hard grade nucleus is a challenging situation. Combined bevel down and up modality could be a good option for the phacoemulsification of the hard cataract as evidenced by the less effective phaco and NET and reduced CDE. Bevel down tip efficiently chops the nucleus, while bevel up emulsifies the nuclear fragments without dissipation of the phaco energy in the anterior chamber. The technique is effective in Asian countries where hard grade of nuclei exists, thus delivering advantage of phacoemulsification.

\section{Acknowledgment}

We thank Dr Avinash Turankar, Associate Professor, Department of Pharmacology, Government Medical College Nagpur, India, for statistical assistance.

\section{Author contributions}

All authors contributed toward conception and design of the study, data acquisition, analysis, and interpretation, drafting and revising the paper, and agree to be accountable for all aspects of the work.

\section{Disclosure}

The authors report no conflicts of interest in this work.

\section{References}

1. Kim EK, Jo KJ, Joo CK. Comparison of tips in coaxial microincision cataract surgery with the bevel-down technique. J Cataract Refract Surg. 2011;37(11):2028-2033.

2. Joshi RS. Primary posterior capsular opacification in Indian rural population undergoing cataract surgery for hypermature senile cataract. Clin Ophthalmol. 2013;7:1605-1608.

3. Khanna R, Pujari S, Sangwan V. Cataract surgery in developing countries. Curr Opin Ophthalmol. 2011;22(1):10-14.

4. Chylack LT Jr, Wolfe JK, Singer DM, et al. The lens opacities classification system III. Arch Ophthalmol. 1993;111(6):831-836.

5. Joshi RS. A single drop $0.5 \%$ proparacaine hydrochloride for uncomplicated clear corneal phacoemulsification. Middle East Afr J Ophthalmol. 2013;20(3):221-224.

6. Haripriya A, Chang DF, Reena M, Shekhar M. Complication rates of phacoemulsification and manual small-incision cataract surgery at Aravind Eye Hospital. J Cataract Refract Surg. 2012;38(8):1360-1369.

7. Lee JS, Hou CH, Yang ML, Kuo JZ, Lin KK. A different approach to assess resident phacoemulsification learning curve: analysis of both completion and complication rates. Eye (Lond). 2009;23(3):683-687.

8. Fine IH, Packer M, Hoffman RS. Power modulations in new phacoemulsification technology: improved outcomes. J Cataract Refract Surg. 2004;30(5):1014-1019.

9. Rekas M, Montés-Micó R, Krix-Jachym K, Kluś A, Stankiewicz A, Ferrer-Blasco T. Comparison of torsional and longitudinal modes using phacoemulsification parameters. J Cataract Refract Surg. 2009; 35(10):1719-1724.

10. Raskin E, Paula JS, Cruz AA, Coelho RP. Effect of bevel position on the corneal endothelium after phacoemulsification. Arq Bras Oftalmol. 2010;73(6):508-510.

11. Park JH, Lee SM, Kwon JW, et al. Ultrasound energy in phacoemulsification: a comparative analysis of phaco-chop and stop-and-chop techniques according to the degree of nuclear density. Ophthalmic Surg Lasers Imaging. 2010;41(2):236-241.

12. Maalej A, Khallouli A, Wathek C, Rannen R. Ozil ${ }^{\circledR}$ versus conventional ultrasound phacoemulsification: a randomized comparative study. Int Ophthalmol. 2015;35(4):565-568.

13. Doors M, Berendschot TT, Touwslager W, Webers CA, Nuijts RM. Phacopower modulation and the risk for postoperative corneal decompensation: a randomized clinical trial. JAMA Ophthalmol. 2013;131(11): 1443-1450.

14. Kim DH, Wee WR, Lee JH, Kim MK. The comparison between torsional and conventional mode phacoemulsification in moderate and hard cataracts. Korean J Ophthalmol. 2010;24(6):336-340.

15. Wang Y, Xia Y, Zeng M, et al. Torsional ultrasound efficiency under different vacuum levels in different degrees of nuclear cataract. J Cataract Refract Surg. 2009;35(11):1941-1945. 
16. Reuschel A, Bogatsch H, Barth T, Wiedemann R. Comparison of endothelial changes and power settings between torsional and longitudinal phacoemulsification. J Cataract Refract Surg. 2010;36(11): 1855-1861.

17. Chen M, Sweeney HW, Luke B, Chen M, Brown M. A retrospective randomized study to compare the energy delivered using CDE with different techniques and $\mathrm{OZil}^{\circledR}$ settings by different surgeons in phacoemulsification. Clin Ophthalmol. 2009;3:401-403.

18. Chen M, Chen M. Comparison of CDE data in phacoemulsification between an open hospital-based ambulatory surgical center and a free-standing ambulatory surgical center. Clin Ophthalmol. 2010;4: 1287-1289.
19. Fakhry MA, El Shazly MI. Torsional ultrasound mode versus combined torsional and conventional ultrasound mode phacoemulsification for eyes with hard cataract. Clin Ophthalmol. 2011;5:973-978.

20. Joo CK, Kim YH. Phacoemulsification with a bevel-down phaco tip: phaco-drill. J Cataract Refract Surg. 1997;23(8):1149-1152.

21. Kohlhaas M, Klemm M, Kammann J, Richard G. Endothelial cell loss secondary to two different phacoemulsification techniques. Ophthalmic Surg Lasers. 1998;29(11):890-895.

22. Joo CK, Kim YH. Phacoemulsification with a bevel-down phaco tip: phaco-drill. J Cataract Refract Surg. 1997;23(8):1149-1152. Comment in: J Cataract Refract Surg. 1998;24(2):147.
Clinical Ophthalmology

\section{Publish your work in this journal}

Clinical Ophthalmology is an international, peer-reviewed journal covering all subspecialties within ophthalmology. Key topics include: Optometry; Visual science; Pharmacology and drug therapy in eye diseases; Basic Sciences; Primary and Secondary eye care; Patien Safety and Quality of Care Improvements. This journal is indexed on

Submit your manuscript here: http://www.dovepress.com/clinical-ophthalmology-journal

\section{Dovepress}

PubMed Central and CAS, and is the official journal of The Society of Clinical Ophthalmology (SCO). The manuscript management system is completely online and includes a very quick and fair peer-review system, which is all easy to use. Visit http://www.dovepress.com/ testimonials.php to read real quotes from published authors. 